\title{
A new constrained mKP hierarchy and the generalized Darboux transformation for the mKP equation with self-consistent sources
}

\author{
Ting Xiao Yunbo Zeng $\dagger$ \\ Department of Mathematical Sciences, Tsinghua University, Beijing 100084, China \\ †Email: yzeng@math.tsinghua.edu.cn
}

\begin{abstract}
The mKP equation with self-consistent sources (mKPESCS) is treated in the framework of the constrained mKP hierarchy. We introduce a new constrained mKP hierarchy which may be viewed as the stationary hierarchy of the mKP hierarchy with self-consistent sources. This offers a natural way to obtain the Lax representation for the mKPESCS. Based on the conjugate Lax pairs, we construct the generalized Darboux transformation with arbitrary functions in time $t$ for the mKPESCS which, in contrast with the Darboux transformation for the mKP equation, provides a non-autoBäcklund transformation between two mKPESCSs with different degrees. The formula for $n$-times repeated generalized Darboux transformation is proposed and enables us to find the rational solutions (including the lump solutions), soliton solutions and the solutions of breather type of the mKPESCS.
\end{abstract}

Keywords: Lax representation; constrained mKP hierarchy; mKP equation with selfconsistent sources(mKPESCS); Darboux transformation(DT); rational solution; soliton solution; solution of breather type

\section{Introduction}

Soliton equations with self-consistent sources (SESCSs) are important models in many fields of physics, such as hydrodynamics, solid state physics, plasma physics, etc. [1-8,15]. 
For example, the nonlinear Schrödinger equation with self-consistent sources represents the nonlinear interaction of an electrostatic high-frequency wave with the ion acoustic wave in a two component homogeneous plasma[8]. The KdV equation with self-consistent sources describes the interaction of long and short capillary-gravity waves[4]. The KP equation with self-consistent sources describes the interaction of a long wave with a short-wave packet propagating on the $\mathrm{x}, \mathrm{y}$ plane at an angle to each other(see [15] and the references therein). Until now, much development has been made in the study of SESCS. For example, in (1+1)dimensional case, some SESCSs such as the KdV, modified KdV, nonlinear Schrödinger, AKNS and Kaup-Newell hierarchies with self-consistent sources were solved by the inverse scattering method [1,2,3,6-10]. Also a type of generalized binary Darboux transformations with arbitrary functions in time $t$ for some $(1+1)$-dimensional SESCSs , which offer a nonauto-Bäcklund transformation between two SESCSs with different degrees of sources, have been constructed and can be used to obtain N-soliton, positon and negaton solution [12-14]. In $(2+1)$-dimensional case, some results to the SESCSs have been obtained. The soliton solution of the KP equation with self-consistent sources (KPESCS) was first found by Mel'nikov $[15,16]$. However, since the explicit time part of the Lax representation of the KPESCS was not found, the method to solve the KPESCS by inverse scattering transformation in $[15,16]$ was quite complicated. In [17], in the framework of the constrained KP hierarchy, we get the Lax representation of the KP equation with self-consistent sources naturally and construct the generalized binary Darboux transformation for it naturally. The KPESCS is also studied by Hirota method in [18].

In this paper, we develop the idea presented in [17] to study the mKP equation with selfconsistent sources. First we give a new constrained mKP hierarchy which may be viewed as the stationary hierarchy of the mKP hierarchy with self-consistent sources. This gives a natural way to find the Lax representation for the mKPESCS. Using the conjugate Lax pairs, we construct the generalized Darboux transformation with arbitrary functions in time $t$ for the mKPESCS. In contrast with the Darboux transformation for the mKP equation which offers a Bäcklund transformation, this transformation provides a non-auto-Bäcklund transformation between two mKPESCSs with different degrees of sources. By this generalized Darboux transformation, some interesting solutions of mKPESCS such as soliton solutions, rational solutions (including lump solutions) and solutions of breather type are obtained.

The paper will be organized as follows. We recall some facts about the mKP hierarchy and mKP equation through the pseudo-differential operator (PDO) formalism in the next section. In section 3, we introduce a new constrained mKP hierarchy and give some examples of equations. In section 4 , we reveal the relation between the mKP hierarchy with self-consistent 
sources and the constrained mKP hierarchy given in the previous section. Then the conjugate Lax pairs of the mKP hierarchy with self-consistent sources can be obtained naturally. Using the conjugate Lax pairs, we can construct the generalized Darboux transformations with arbitrary functions in time for the mKPESCS. In Section 5, the n-times repeated generalized Darboux transformation will be constructed by which some interesting solutions for the mKPESCS are obtained in section 6.

\section{The mKP hierarchy and the mKP equation}

Let us consider the following pseudo-differential operator(PDO)

$$
L=L_{m K P}=\partial+V+V_{1} \partial^{-1}+V_{2} \partial^{-2}+\ldots,
$$

where $\partial$ denotes $\frac{\partial}{\partial x}$, and $V, V_{j}, j=1, \ldots$ are functions. Denote $B_{m}=\left(L^{m}\right)_{\geq 1}$ for $\forall m \in N$ where $\left(L^{m}\right)_{\geq 1}$ represents the projection of $L^{m}$ to its differential part whose order is more than 1. Then the mKP hierarchy is defined as [19]

$$
L_{t_{k}}=\left[B_{k}, L\right], k \geq 1
$$

or the equivalent form

$$
\left(L^{n}\right)_{t_{k}}=\left[B_{k}, L^{n}\right], n, k \geq 1 .
$$

The mKP hierarchy (2.2) can also be written in the zero-curvature form

$$
\left(B_{n}\right)_{t_{m}}-\left(B_{m}\right)_{t_{n}}+\left[B_{n}, B_{m}\right]=0, \quad n, m \geq 2
$$

The equation (2.4) has a pair of conjugate Lax pairs as follows

$$
\begin{aligned}
& \psi_{1, t_{m}}=\left(B_{m} \psi_{1}\right), \\
& \psi_{1, t_{n}}=\left(B_{n} \psi_{1}\right),
\end{aligned}
$$

and

$$
\begin{aligned}
& \psi_{2, t_{m}}=\left(\tilde{B}_{m} \psi_{2}\right), \\
& \psi_{2, t_{n}}=\left(\tilde{B}_{n} \psi_{2}\right),
\end{aligned}
$$

where $\tilde{B}_{k}=-\left(\partial B_{k} \partial^{-1}\right)^{*}, k \geq 2$. We make a convention that for any operator $P$ and a function $f,(P f)$ means that the operator $P$ acts on $f$ while $P f$ means the product of $P$ 
and $f$. It is easy to see that $\tilde{B}_{k}$ are also differential operators. When $n=2, m=3$ we get the mKP equation as follows

$$
4 V_{t_{3}}-V_{x x x}+6 V^{2} V_{x}-3\left(D^{-1} V_{t_{2} t_{2}}\right)-6 V_{x}\left(D^{-1} V_{t_{2}}\right)=0
$$

where $D D^{-1}=D^{-1} D=1$. Set

$$
u=-V, \quad t=-\frac{1}{4} t_{3}, \quad y=\alpha t_{2}
$$

the mKP equation will be written as

$$
u_{t}-6 u^{2} u_{x}+u_{x x x}+3 \alpha^{2}\left(D^{-1} u_{y y}\right)-6 \alpha u_{x}\left(D^{-1} u_{y}\right)=0
$$

which is called the mKPI equation when $\alpha=i$ and mKPII equation when $\alpha=1$. From (2.5) and (2.6), we will get the conjugate Lax pairs of (2.9) respectively as follows

$$
\begin{gathered}
\alpha \psi_{1, y}=\psi_{1, x x}-2 u \psi_{1, x}, \\
\psi_{1, t}=\left(A_{1}(u) \psi_{1}\right), \quad A_{1}(u)=-4 \partial^{3}+12 u \partial^{2}-6\left(-u_{x}+u^{2}-\alpha D^{-1} u_{y}\right) \partial,
\end{gathered}
$$

and

$$
\begin{gathered}
\alpha \psi_{2, y}=-\psi_{2, x x}-2 u \psi_{2, x}, \\
\psi_{2, t}=\left(A_{2}(u) \psi_{2}\right), \quad A_{2}(u)=-4 \partial^{3}-12 u \partial^{2}-6\left(u_{x}+u^{2}-\alpha D^{-1} u_{y}\right) \partial,
\end{gathered}
$$

It is known that the system (2.10) is covariant w.r.t. the following transformations [20]

$$
\begin{aligned}
& \psi_{1}[1]=\psi_{1}-f_{1} \frac{\int \psi_{1, x} g_{1} \mathrm{~d} x+C_{2}}{\int f_{1, x} g_{1} \mathrm{~d} x-C_{1}}, \\
& u[1]=u+\partial_{x} \ln \frac{\int g_{1, x} f_{1} \mathrm{~d} x+C_{1}}{\int f_{1, x} g_{1} \mathrm{~d} x-C_{1}},
\end{aligned}
$$

while the system (2.11) is covariant w.r.t.

$$
\begin{aligned}
& \psi_{2}[1]=\psi_{2}-g_{1} \frac{\int \psi_{2, x} f_{1} \mathrm{~d} x+C_{2}}{\int g_{1, x} f_{1} \mathrm{~d} x+C_{1}}, \\
& u[1]=u+\partial_{x} \ln \frac{\int g_{1, x} f_{1} \mathrm{~d} x+C_{1}}{\int f_{1, x} g_{1} \mathrm{~d} x-C_{1}},
\end{aligned}
$$

where $f_{1}, g_{1}$ are solutions of (2.10) and (2.11) respectively and $C_{1}, C_{2}$ are arbitrary constants. We point out that throughout the paper, the integral operation $\int f_{1} f_{2} \mathrm{~d} x$ means $\int_{-\infty}^{x} f_{1} f_{2} \mathrm{~d} x$ or $-\int_{x}^{\infty} f_{1} f_{2} \mathrm{~d} x$ and contains no arbitrary function of $y$ and $t$, only numerical constant if we 
impose some suitable boundary condition on the integrand functions $f_{1}$ and $f_{2}$ at $x=-\infty$ or $x=\infty$. Substituting (2.12a) (with $C_{2}=0, C_{1}=C$ ) into $(2.10 \mathrm{~b})$, we will get the following identity

$$
\begin{aligned}
& \left(A_{1}(u[1]) \psi_{1}[1]\right) \\
= & \left(\psi_{1}-f_{1} \frac{\int \psi_{1, x} g_{1} \mathrm{~d} x}{\int f_{1, x} g_{1} \mathrm{~d} x-C}\right)_{t} \\
= & \psi_{1, t}-f_{1, t} \frac{\int \psi_{1, x} g_{1} \mathrm{~d} x}{\int f_{1, x} g_{1} \mathrm{~d} x-C}-f_{1} \frac{\int\left(\psi_{1, x} g_{1, t}+\psi_{1, x t} g_{1}\right) \mathrm{d} x\left(\int f_{1, x} g_{1} \mathrm{~d} x-C\right)-\left(\int \psi_{1, x} g_{1} \mathrm{~d} x\right)\left[\int\left(g_{1, t} f_{1, x}+g_{1} f_{1, x t}\right) \mathrm{d} x\right]}{\left(\int f_{1, x} g_{1} \mathrm{~d} x-C\right)^{2}} \\
= & \left(A_{1}(u) \psi_{1}\right)-\left(A_{1}(u) f_{1}\right) \frac{\int \psi_{1, x} g_{1} \mathrm{~d} x}{\int f_{1, x} g_{1} \mathrm{~d} x-C} \\
& -f_{1} \frac{\left[\int\left(\psi_{1, x}\left(A_{2}(u) g_{1}\right)+\left(A_{1}(u) \psi_{1}\right)_{x} g_{1}\right) \mathrm{d} x\right]\left(\int f_{1, x} g_{1} \mathrm{~d} x-C\right)-\left(\int \psi_{1, x} g_{1} \mathrm{~d} x\right)\left[\int\left(\left(A_{2}(u) g_{1}\right) f_{1, x}+g_{1}\left(A_{1}(u) f_{1}\right)_{x}\right) \mathrm{d} x\right]}{\left(\int f_{1, x} g_{1} \mathrm{~d} x-C\right)^{2}}
\end{aligned}
$$

\section{A new constraint of the mKP hierarchy}

In [19], W.Oevel and W.Strampp have studied the constraint of the PDO $L(2.1)$ as

$$
L^{n}=\left(L^{n}\right)_{\geq 1}+v_{0}+\partial^{-1} \psi
$$

from which we will get the Kaup-Broer hierarchy when $n=1$. Here we consider a new constraint as follows

$$
L^{n}=\left(L^{n}\right)_{\geq 1}+q \partial^{-1} r \partial
$$

where $q, r$ satisfy that

$$
q_{t_{k}}=\left(B_{k} q\right), \quad r_{t_{k}}=\left(\tilde{B}_{k} r\right),
$$

and $B_{k}=\left(\left(L^{n}\right)^{\frac{k}{n}}\right)_{\geq 1}=\left[\left(\left(L^{n}\right)_{\geq 1}+q \partial^{-1} r \partial\right)^{\frac{k}{n}}\right]_{\geq 1}$.

Then a new $n$-constrained mKP hierarchy will be obtained as

$$
\begin{gathered}
\left(L^{n}\right)_{t_{k}}=\left[\left(L^{k}\right)_{\geq 1}, L^{n}\right]=\left[B_{k}, L^{n}\right], \\
q_{t_{k}}=\left(B_{k} q\right), \\
r_{t_{k}}=\left(\tilde{B}_{k} r\right),
\end{gathered}
$$

First, we will prove that the constraint (3.2) together with the condition (3.3) is compatible with the mKP hierarchy (2.2). The following formulas for PDO will be useful in the proof and we list them below,

$$
\begin{gathered}
\left(\Lambda^{*}\right)_{0}=\operatorname{res}\left(\partial^{-1} \Lambda\right), \quad(\Lambda)_{0}=\operatorname{res}\left(\Lambda \partial^{-1}\right), \quad\left(\Lambda \partial^{-1}\right)_{<0}=(\Lambda)_{0} \partial^{-1}+(\Lambda)_{<0} \partial^{-1} \\
\left(P q \partial^{-1} r\right)_{<0}=(P q) \partial^{-1} r, \quad\left(q \partial^{-1} r P\right)_{<0}=q \partial^{-1}\left(P^{*} r\right)
\end{gathered}
$$

where $\Lambda$ is an arbitrary $\mathrm{PDO}$ and $\mathrm{P}$ differential operator. $(A)_{0}$ denote the zero order term for a $\operatorname{PDO} A$. 
Theorem 3.1 The constraint (3.2) together with the condition (3.3) is compatible with the $m K P$ hierarchy (2.2).

Proof: We need to prove the following identity

$$
\begin{aligned}
& \left(q \partial^{-1} r \partial\right)_{t_{k}}=\left[B_{k}, L^{n}\right]_{\leq 0}=\left[B_{k}, q \partial^{-1} r \partial\right]_{\leq 0}, \\
& \text { the 1.h.s. of }(3.6)=q_{t_{k}} \partial^{-1} r \partial+q \partial^{-1} r_{t_{k}} \partial \\
& =\left(B_{k} q\right) \partial^{-1} r \partial+q \partial^{-1}\left(\tilde{B}_{k} r\right) \partial \\
& \triangleq l_{1}+l_{2} \\
& \text { the r.h.s. of }(3.6)=\left(B_{k} q \partial^{-1} r \partial\right)_{\leq 0}-\left(q \partial^{-1} r \partial B_{k}\right)_{\leq 0} \\
& \triangleq r_{1}-r_{2} \\
& \left(l_{1}\right)_{0}=\left(\left(B_{k} q\right) \partial^{-1} r \partial\right)_{0}=\left(B_{k} q\right) r, \quad\left(l_{2}\right)_{0}=\left(q \partial^{-1}\left(\tilde{B}_{k} r\right) \partial\right)_{0}=q\left(\tilde{B}_{k} r\right), \\
& \left(r_{1}\right)_{0}=\operatorname{res}\left[\partial^{-1}\left(r_{1}\right)^{*}\right]=\operatorname{res}\left[\partial^{-1}\left(\partial r \partial^{-1} q B_{k}^{*}\right)\right]=\operatorname{res}\left(r \partial^{-1} q B_{k}^{*}\right) \\
& =\operatorname{res}\left(r \partial^{-1} q B_{k}^{*}\right)_{<0}=\operatorname{res}\left(r \partial^{-1}\left(B_{k} q\right)\right)=r\left(B_{k} q\right) \text {, } \\
& \left(r_{2}\right)_{0}=\left(q \partial^{-1} r \partial B_{k}\right)_{0}=\operatorname{res}\left[q \partial^{-1} r \partial B_{k} \partial^{-1}\right]=\operatorname{res}\left[q \partial^{-1} r\left(\partial B_{k} \partial^{-1}\right)\right] \\
& =\operatorname{res}\left[q \partial^{-1}\left(\left(\partial B_{k} \partial^{-1}\right)^{*} r\right)\right]=q\left(\left(\partial B_{k} \partial^{-1}\right)^{*} r\right)=-q\left(\tilde{B}_{k} r\right) \text {, }
\end{aligned}
$$

So

$$
\begin{gathered}
\left(l_{1}\right)_{0}+\left(l_{2}\right)_{0}=\left(r_{1}\right)_{0}-\left(r_{2}\right)_{0} . \\
\left(l_{1}\right)_{<0}=\left(\left(B_{k} q\right) \partial^{-1} r \partial\right)_{<0}=-\left(B_{k} q\right) \partial^{-1} r_{x}, \\
\left(l_{2}\right)_{<0}=\left(q \partial^{-1}\left(\tilde{B}_{k} r\right) \partial\right)_{<0}=-q \partial^{-1}\left[\partial\left(\left(\tilde{B}_{k} r\right)\right)\right]=q \partial^{-1}\left[\partial \partial^{-1}\left(B_{k}^{*} \partial r\right)\right]=q \partial^{-1}\left(B_{k}^{*} r_{x}\right),
\end{gathered}
$$

By the last formula of (3.5a), we have

$$
\left(r_{1} \partial^{-1}\right)_{<0}=\left(B_{k} q \partial^{-1} r\right)_{<0}=\left(r_{1}\right)_{0} \partial^{-1}+\left(r_{1}\right)_{<0} \partial^{-1}
$$

i.e.

$$
\left(B_{k} q\right) \partial^{-1} r=\left(r_{1}\right)_{0} \partial^{-1}+\left(r_{1}\right)_{<0} \partial^{-1} .
$$

Multiplying $\partial$ on the right and taking the negative part of both sides of the above identity, we get

$$
\left(\left(B_{k} q\right) \partial^{-1} r \partial\right)_{<0}=\left(r_{1}\right)_{<0}
$$

So

$$
\begin{gathered}
\left(r_{1}\right)_{<0}=-\left(B_{k} q\right) \partial^{-1} r_{x} \\
\left(r_{2}\right)_{<0}=\left(q \partial^{-1} r \partial B_{k}\right)_{<0}=q \partial^{-1}\left(\partial B_{k}\right)^{*}(r)=-q \partial^{-1}\left(B_{k}^{*} r_{x}\right)
\end{gathered}
$$


So we have

$$
\left(l_{1}\right)_{<0}+\left(l_{2}\right)_{<0}=\left(r_{1}\right)_{<0}-\left(r_{2}\right)_{<0}
$$

From (3.12) and (3.17), we can see (3.6) holds.

This completes the proof.

We give some examples below.

(a) 1-constraint $(n=1)$.

Here

$$
L=\partial+q \partial^{-1} r \partial .
$$

So

$$
\begin{gathered}
V=q r, \quad V_{1}=-q r_{x}, \quad \ldots \\
B_{2}=\left(L^{2}\right)_{\geq 1}=\partial^{2}+2 q r \partial, \quad \tilde{B}_{2}=-\left(\partial B_{2} \partial^{-1}\right)^{*}=-\partial^{2}+2 q r \partial, \\
B_{3}=\left(L^{3}\right)_{\geq 1}=\partial^{3}+3 q r \partial^{2}+\left(3 q^{2} r^{2}+3 q_{x} r\right) \partial, \\
\tilde{B}_{3}=-\left(\partial B_{3} \partial^{-1}\right)^{*}=\partial^{3}-3 q r \partial^{2}+\left(3 q^{2} r^{2}-3 q r_{x}\right) \partial, \quad \ldots
\end{gathered}
$$

The first two equations of the 1-constrained hierarchy are

$$
\begin{gathered}
q_{t_{2}}=q_{x x}+2 q r q_{x}, \\
r_{t_{2}}=-r_{x x}+2 q r r_{x},
\end{gathered}
$$

and

$$
\begin{aligned}
& q_{t_{3}}=q_{x x x}+3 q r q_{x x}+\left(3 q^{2} r^{2}+3 q_{x} r\right) q_{x}, \\
& r_{t_{3}}=r_{x x x}-3 q r r_{x x}+\left(3 q^{2} r^{2}-3 q r_{x}\right) r_{x} .
\end{aligned}
$$

Equation (3.20) is the generalized NS equation with derivative coupling given by Chen et al $[21,22]$. The constrained hierarchy is also studied in [23].

(b) 2-constraint $(n=2)$.

Here

$$
L^{2}=\partial^{2}+2 V \partial+q \partial^{-1} r \partial .
$$

from which we find

$$
\begin{gathered}
V_{1}=q r-V_{x}-V^{2}, \quad \ldots \\
B_{2}=\left(L^{2}\right)_{\geq 1}=\partial^{2}+2 V \partial, \quad \tilde{B}_{2}=-\left(\partial B_{2} \partial^{-1}\right)^{*}=-\partial^{2}+2 V \partial, \\
B_{3}=\left(L^{3}\right)_{\geq 1}=\partial^{3}+3 V \partial^{2}+3 q r \partial, \quad \tilde{B}_{3}=-\left(\partial B_{3} \partial^{-1}\right)^{*}=\partial^{3}-3 V \partial^{2}+\left(3 q r-3 V_{x}\right) \partial, \quad \ldots
\end{gathered}
$$


The first two equations of the 2-constrained hierarchy are

$$
\begin{gathered}
V_{t_{2}}=(q r)_{x}, \\
q_{t_{2}}=q_{x x}+2 V q_{x}, \\
r_{t_{2}}=-r_{x x}+2 V r_{x} .
\end{gathered}
$$

and

$$
\begin{gathered}
V_{t_{3}}=V_{x x x}+3 V V_{x x}+6 V^{2} V_{x}+3 q r V_{x}-\frac{3}{2}\left(q r_{x}\right)_{x} \\
q_{t_{3}}=q_{x x x}+3 V q_{x x}+3 q r q_{x} \\
r_{t_{3}}=r_{x x x}-3 V r_{x x}+\left(3 q r-3 V_{x}\right) r_{x}
\end{gathered}
$$

(c) 3-constraint $(n=3)$.

Here

$$
L^{3}=\partial^{3}+3 V \partial^{2}+3\left(V^{2}+V_{x}+V_{1}\right) \partial+q \partial^{-1} r \partial .
$$

The first equation of the 3-constrained hierarchy is

$$
\begin{gathered}
V_{t_{2}}=V_{x x}+2 V_{1, x}+2 V V_{x}, \\
3 V_{1, t_{2}}=-2 V_{x x x}-6 V V_{x x}-6 V^{2} V_{x}-6 V_{x}^{2}-3 V_{1, x x}-6 V V_{1, x}-6 V_{1} V_{x}+2(q r)_{x}, \\
q_{t_{2}}=q_{x x}+2 V q_{x} \\
r_{t_{2}}=-r_{x x}+2 V r_{x} .
\end{gathered}
$$

Eliminating $V_{1}$ from the above equation, we get

$$
\begin{gathered}
\frac{1}{2} V_{x x x}+\frac{3}{2} D^{-1}\left(V_{y y}\right)+3\left(D^{-1} V_{y}\right) V_{x}-3 V^{2} V_{x}-2(q r)_{x}=0 \\
q_{t_{2}}=q_{x x}+2 V q_{x} \\
r_{t_{2}}=-r_{x x}+2 V r_{x} .
\end{gathered}
$$

\section{The mKP equation with self-consistent sources and its generalized Darboux transformation}

If generalizing the constraint (3.2) to

$$
L^{n}=\left(L^{n}\right)_{\geq 1}+\sum_{i=1}^{N} q_{i} \partial^{-1} r_{i} \partial
$$


where

$$
q_{i, t_{k}}=\left(B_{k} q_{i}\right), \quad r_{i, t_{k}}=\left(\tilde{B}_{k} r_{i}\right),
$$

and adding the term $\left(B_{k}\right)_{t_{n}}$ to the right hand side of $(3.4 \mathrm{a})$, we can define the mKP hierarchy with self-consistent sources as follows

$$
\begin{gathered}
\left(B_{k}\right)_{t_{n}}-\left(L^{n}\right)_{t_{k}}+\left[B_{k}, L^{n}\right]=0 \\
q_{i, t_{k}}=\left(B_{k} q_{i}\right) \\
r_{i, t_{k}}=\left(\tilde{B}_{k} r_{i}\right)
\end{gathered}
$$

So if the variable " $t_{n}$ " is viewed as the evolution variable, the $n$-constrained mKP hierarchy may be regarded as the stationary hierarchy of the mKP hierarchy with self-consistent sources. Under the condition (4.3b) and (4.3c), we naturally get the conjugate Lax pairs of (4.3a) as follows

$$
\begin{gathered}
\psi_{1, t_{k}}=\left(B_{k} \psi_{1}\right) \\
\psi_{1, t_{n}}=\left(L^{n} \psi_{1}\right)=\left(B_{n} \psi_{1}\right)+\sum_{i=1}^{N} q_{i} \int r_{i} \psi_{1, x} \mathrm{~d} x
\end{gathered}
$$

and

$$
\begin{gathered}
\psi_{2, t_{k}}=\left(\tilde{B}_{k} \psi_{2}\right) \\
\psi_{2, t_{n}}=\left(\tilde{L}^{n} \psi_{2}\right)=\left(\tilde{B}_{n} \psi_{2}\right)-\left(\left[\partial\left(\sum_{i=1}^{N} q_{i} \partial^{-1} r_{i} \partial\right) \partial^{-1}\right]^{*} \psi_{2}\right)=\left(\tilde{B}_{n} \psi_{2}\right)-\sum_{i=1}^{N} r_{i} \int q_{i} \psi_{2, x} \mathrm{~d} x
\end{gathered}
$$

When $n=3, k=2$, under the transformation (2.8) and setting

$$
\Phi_{i}=r_{i}, \quad \Psi_{i}=q_{i}
$$

we will get the mKP equation with self-consistent sources (mKPESCS) and its conjugate Lax pairs respectively from (4.3),(4.4) and (4.5).

The mKPESCS is

$$
\begin{gathered}
u_{t}+u_{x x x}+3 \alpha^{2} D^{-1}\left(u_{y y}\right)-6 \alpha D^{-1}\left(u_{y}\right) u_{x}-6 u^{2} u_{x}+4 \sum_{i=1}^{N}\left(\Psi_{i} \Phi_{i}\right)_{x}=0 \\
\alpha \Psi_{i, y}=\Psi_{i, x x}-2 u \Psi_{i, x} \\
\alpha \Phi_{i, y}=-\Phi_{i, x x}-2 u \Phi_{i, x}
\end{gathered}
$$


which is called the mKPIESCS when $\alpha=i$ and mKPIIESCS when $\alpha=1$. Under the condition (4.6b) and (4.6c), the conjugate Lax pairs for (4.6a) are

$$
\begin{gathered}
\alpha \psi_{1, y}=\psi_{1, x x}-2 u \psi_{1, x}, \\
\psi_{1, t}=\left(A_{1}(u) \psi_{1}\right)+T_{N}^{1}(\Psi, \Phi) \psi_{1}, \quad T_{N}^{1}(\Psi, \Phi) \psi_{1}=-4 \sum_{i=1}^{N} \Psi_{i} \int \Phi_{i} \psi_{1, x} \mathrm{~d} x,
\end{gathered}
$$

and

$$
\begin{gathered}
\alpha \psi_{2, y}=-\psi_{2, x x}-2 u \psi_{2, x} \\
\psi_{2, t}=\left(A_{2}(u) \psi_{2}\right)+T_{N}^{2}(\Psi, \Phi) \psi_{2}, \quad T_{N}^{2}(\Psi, \Phi) \psi_{2}=4 \sum_{i=1}^{N} \Phi_{i} \int \Psi_{i} \psi_{2, x} \mathrm{~d} x
\end{gathered}
$$

For the system (4.7), we can construct the following Darboux transformation.

Theorem 4.1 Assume $u, \Phi_{1}, \ldots, \Phi_{N}, \Psi_{1}, \ldots, \Psi_{N}$ be a solution of the mKPESCS (4.6) and $f_{1}, g_{1}$ satisfy (4.7) and (4.8) respectively, then the system (4.7) has the following Darboux transformation

$$
\begin{gathered}
\psi_{1}[1]=\psi_{1}-f_{1} \frac{\int \psi_{1, x} g_{1} \mathrm{~d} x}{\int f_{1, x} g_{1} \mathrm{~d} x-C}, \\
u[1]=u+\partial_{x} \ln \frac{\int g_{1, x} f_{1} \mathrm{~d} x+C}{\int f_{1, x} g_{1} \mathrm{~d} x-C}=u+\frac{g_{1, x} f_{1}}{\int g_{1, x} f_{1} \mathrm{~d} x+C}-\frac{f_{1, x} g_{1}}{\int f_{1, x} g_{1} \mathrm{~d} x-C}, \\
\Psi_{j}[1]=\Psi_{j}-f_{1} \frac{\int \Psi_{i, x} g_{1} \mathrm{~d} x}{\int f_{1, x} g_{1} \mathrm{~d} x-C}, \\
\Phi_{j}[1]=\Phi_{j}-g_{1} \frac{\int f_{1} \Phi_{j, x} \mathrm{~d} x}{\int f_{1} g_{1, x} \mathrm{~d} x+C}, \quad j=1, \ldots, N .
\end{gathered}
$$

where $C$ is an arbitrary constant.

Proof: It is obvious that $u[1], \psi_{1}[1], \Phi_{i}[1], \Psi_{i}[1], i=1, \ldots, N$ satisfy $(4.6 \mathrm{~b}),(4.6 \mathrm{c})$ and (4.7a). So we only need to prove that $(4.7 \mathrm{~b})$ holds, i.e., to prove the following equality

$$
\psi_{1}[1]_{t}=\left(A_{1}(u[1]) \psi_{1}[1]\right)+T_{N}^{1}(\Psi[1], \Phi[1]) \psi_{1}[1]
$$

Using (4.7b), we have

$$
\begin{aligned}
\psi_{1}[1]_{t}= & \left(A_{1}(u) \psi_{1}\right)+T_{N}^{1}(\Psi, \Phi) \psi_{1}-\left(\left(A_{1}(u) f_{1}\right)+T_{N}^{1}(\Psi, \Phi) f_{1}\right) \frac{\int \psi_{1, x} g_{1} \mathrm{~d} x}{\int f_{1, x} g_{1} \mathrm{~d} x-C} \\
& -f_{1} \frac{\int\left(\left(A_{2}(u) g_{1}\right)+T_{N}^{2}(\Psi, \Phi) g_{1}\right) \psi_{1, x} \mathrm{~d} x+\int g_{1}\left(\left(A_{1}(u) \psi_{1}\right)+T_{N}^{1}(\Psi, \Phi) \psi_{1}\right)_{x} \mathrm{~d} x}{\int f_{1, x} g_{1} \mathrm{~d} x-C} \\
& +f_{1}\left(\int g_{1} \psi_{1, x} \mathrm{~d} x\right) \frac{\int\left(\left(A_{2}(u) g_{1}\right)+T_{N}^{2}(\Psi, \Phi) g_{1}\right) f_{1, x} \mathrm{~d} x+\int g_{1}\left(\left(A_{1}(u) f_{1}\right)+T_{N}^{1}(\Psi, \Phi) f_{1}\right)_{x} \mathrm{~d} x}{\left(\int f_{1, x} g_{1} \mathrm{~d} x-C\right)^{2}}
\end{aligned}
$$


It is easy to verify that (2.14) still holds now. So we only need to prove the following identity

$$
\begin{aligned}
& T_{N}^{1}(\Psi[1], \Phi[1]) \psi_{1}[1] \\
= & T_{N}^{1}(\Psi, \Phi) \psi_{1}-T_{N}^{1}(\Psi, \Phi) f_{1} \frac{\int \psi_{1, x} g_{1} \mathrm{~d} x}{\int f_{1, x} g_{1} \mathrm{~d} x-C}-f_{1} \frac{\int T_{N}^{2}(\Psi, \Phi) g_{1} \psi_{1, x} \mathrm{~d} x+\int g_{1}\left(T_{N}^{1}(\Psi, \Phi) \psi_{1}\right)_{x} \mathrm{~d} x}{\int f_{1, x} g_{1} \mathrm{~d} x-C} \\
& +f_{1}\left(\int g_{1} \psi_{1, x} \mathrm{~d} x\right) \frac{\int T_{N}^{2}(\Psi, \Phi) g_{1} f_{1, x} \mathrm{~d} x+\int g_{1}\left(T_{N}^{1}(\Psi, \Phi) f_{1}\right)_{x} \mathrm{~d} x}{\left(\int f_{1, x} g_{1} \mathrm{~d} x-C\right)^{2}}
\end{aligned}
$$

By substituting the expression of $T_{N}^{1}$ and $T_{N}^{2}$ in (4.7b) and (4.8b), we find

the r.h.s. of (4.12)

$$
\begin{aligned}
= & -4 \sum_{j=1}^{N} \Psi_{j} \int \Phi_{j} \psi_{1, x} \mathrm{~d} x+4 f_{1} \sum_{j=1}^{N} \frac{\left(\int g_{1} \Psi_{j, x} \mathrm{~d} x\right)\left(\int \Phi_{j} \psi_{1, x} \mathrm{~d} x\right)}{\int f_{1, x} g_{1} \mathrm{~d} x-C} \\
& +4 \sum_{j=1}^{N}\left(\Psi_{j}-f_{1} \frac{\int g_{1} \Psi_{j, x} \mathrm{~d} x}{\int f_{1, x} g_{1} \mathrm{~d} x-C}\right) \frac{\left(\int g_{1} \psi_{1, x} \mathrm{~d} x\right)\left(\int \Phi_{j} f_{1, x} \mathrm{~d} x\right)}{\int f_{1, x} g_{1} \mathrm{~d} x-C} .
\end{aligned}
$$

Then using (4.9) and (4.7b), we can show that

the l.h.s. of (4.12)

$$
\begin{aligned}
= & -4 \sum_{j=1}^{N} \Psi_{j} \int \Phi_{j} \psi_{1, x} \mathrm{~d} x+4 f_{1} \sum_{j=1}^{N} \frac{\left(\int g_{1} \Psi_{j, x} \mathrm{~d} x\right)\left(\int \Phi_{j} \psi_{1, x} \mathrm{~d} x\right)}{\int f_{1, x} g_{1} \mathrm{~d} x-C} \\
& +4 \sum_{j=1}^{N}\left(\Psi_{j}-f_{1} \frac{\int g_{1} \Psi_{j, x} \mathrm{~d} x}{\int f_{1, x} g_{1} \mathrm{~d} x-C}\right) \frac{\left(\int g_{1} \psi_{1, x} \mathrm{~d} x\right)\left(\int \Phi_{j} f_{1, x} \mathrm{~d} x\right)}{\int f_{1, x} g_{1} \mathrm{~d} x-C} \\
= & \text { the r.h.s. of }(4.12) .
\end{aligned}
$$

This completes the proof.

If $C$ is replaced by $C(t)$, an arbitrary function in time $t$ in (4.9), then (4.6b),(4.6c) and (4.7a) are also covariant w.r.t. (4.9), but (4.7b) is not covariant w.r.t. (4.9) any longer. In fact, we have the following theorem.

Theorem 4.2 Given $u, \Psi_{1}, \ldots, \Psi_{N}, \Phi_{1}, \ldots, \Phi_{N}$ a solution of the mKPESCS (4.6) and let $f_{1}$ and $g_{1}$ be solutions of the system (4.7) and (4.8) respectively, then the transformation with $C(t)$ (an arbitrary function in $t$ ) defined by

$$
\begin{gathered}
\psi_{1}[1]=\psi_{1}-f_{1} \frac{\int \psi_{1, x} g_{1} \mathrm{~d} x}{\int f_{1, x} g_{1} \mathrm{~d} x-C(t)}, \\
u[1]=u+\partial_{x} \ln \frac{\int g_{1, x} f_{1} \mathrm{~d} x+C(t)}{\int f_{1, x} g_{1} \mathrm{~d} x-C(t)}=u+\frac{g_{1, x} f_{1}}{\int g_{1, x} f_{1} \mathrm{~d} x+C(t)}-\frac{f_{1, x} g_{1}}{\int f_{1, x} g_{1} \mathrm{~d} x-C(t)}, \\
\Psi_{j}[1]=\Psi_{j}-f_{1} \frac{\int \Psi_{i, x} g_{1} \mathrm{~d} x}{\int f_{1, x} g_{1} \mathrm{~d} x-C(t)}, \\
\Phi_{j}[1]=\Phi_{j}-g_{1} \frac{\int f_{1} \Phi_{j, x} \mathrm{~d} x}{\int f_{1} g_{1, x} \mathrm{~d} x+C(t)}, \quad j=1, \ldots, N,
\end{gathered}
$$


and

$$
\Psi_{N+1}[1]=-\frac{1}{2} \frac{\sqrt{\dot{C}(t)} f_{1}}{\int f_{1, x} g_{1} \mathrm{~d} x-C(t)}, \quad \Phi_{N+1}[1]=\frac{1}{2} \frac{\sqrt{\dot{C}(t)} g_{1}}{\int g_{1, x} f_{1} \mathrm{~d} x+C(t)},
$$

transforms (4.6b),(4.6c) and (4.7) respectively into

$$
\begin{gathered}
\alpha \Psi_{i}[1]_{y}=\Psi_{i}[1]_{x x}-2 u[1] \Psi_{i}[1]_{x}, \\
\alpha \Phi_{i}[1]_{y}=-\Phi_{i}[1]_{x x}-2 u[1] \Phi_{i}[1]_{x}, \quad i=1, \ldots, N+1 \\
\alpha \psi_{1}[1]_{y}=\psi_{1}[1]_{x x}-2 u[1] \psi_{1}[1]_{x}, \\
\psi_{1}[1]_{t}=A_{1}(u[1]) \psi_{1}[1]+T_{N+1}^{1}(\Psi[1], \Phi[1]) \psi_{1}[1] .
\end{gathered}
$$

So $u[1], \Psi_{i}[1], \Phi_{i}[1], i=1, \ldots, N+1$ is a new solution of the mKPESCS (4.6) with degree $N+1$.

Proof: Equations (4.16a), (4.16b) and (4.16c) hold obviously. We only need to prove (4.16d). Substituting (4.15a) into the left hand side of $(4.16 \mathrm{~d})$ and using the result of the previous theorem, we have

$$
\psi_{1}[1]_{t}=\left(\psi_{1}-f_{1} \frac{\int \psi_{1, x} g_{1} \mathrm{~d} x}{\int f_{1, x} g_{1} \mathrm{~d} x-C(t)}\right)_{t}=A_{1}(u[1]) \psi_{1}[1]+T_{N}^{1}(\Psi[1], \Phi[1]) \psi_{1}[1]-\frac{\dot{C}(t) f_{1} \int \psi_{1, x} g_{1} \mathrm{~d} x}{\left(\int f_{1, x} g_{1} \mathrm{~d} x-C(t)\right)^{2}},
$$

So we only need to prove

$$
-4 \Psi_{N+1}[1] \int \Phi_{N+1}[1] \psi_{1, x}[1] \mathrm{d} x=-\frac{\dot{C}(t) f_{1} \int \psi_{1, x} g_{1} \mathrm{~d} x}{\left(\int f_{1, x} g_{1} \mathrm{~d} x-C(t)\right)^{2}},
$$

i.e.

$\frac{\dot{C}(t) f_{1}}{\int f_{1, x} g_{1} \mathrm{~d} x-C(t)} \int \frac{g_{1}}{\int f_{1} g_{1, x} \mathrm{~d} x+C(t)}\left(\psi_{1}-f_{1} \frac{\int \psi_{1, x} g_{1} \mathrm{~d} x}{\int f_{1, x} g_{1} \mathrm{~d} x-C(t)}\right)_{x} \mathrm{~d} x=-\frac{\dot{C}(t) f_{1} \int \psi_{1, x} g_{1} \mathrm{~d} x}{\left(\int f_{1, x} g_{1} \mathrm{~d} x-C(t)\right)^{2}}$, i.e., to prove

$$
\int \frac{g_{1}}{\int f_{1} g_{1, x} \mathrm{~d} x+C(t)}\left(\psi_{1}-f_{1} \frac{\int \psi_{1, x} g_{1} \mathrm{~d} x}{\int f_{1, x} g_{1} \mathrm{~d} x-C(t)}\right)_{x} \mathrm{~d} x=-\frac{\int \psi_{1, x} g_{1} \mathrm{~d} x}{\int f_{1, x} g_{1} \mathrm{~d} x-C(t)},
$$

the 1.h.s of (4.18)

$$
\begin{aligned}
& =\int \frac{g_{1}}{\int f_{1} g_{1, x} \mathrm{~d} x+C(t)}\left(\psi_{1, x}-\frac{f_{1, x} \int g_{1} \psi_{1, x} \mathrm{~d} x+f_{1} g_{1} \psi_{1, x}}{\int f_{1, x} g_{1} \mathrm{~d} x-C(t)}+f_{1} g_{1} f_{1, x} \frac{\int g_{1} \psi_{1, x} \mathrm{~d} x}{\left(\int f_{1, x} g_{1} \mathrm{~d} x-C(t)\right)^{2}}\right) \mathrm{d} x \\
& =\int\left[-\frac{g_{1} \psi_{1, x}}{\int f_{1, x} g_{1} \mathrm{~d} x-C(t)}+\frac{g_{1} f_{1, x} \int g_{1} \psi_{1, x} \mathrm{~d} x}{\left(\int f_{1, x} g_{1} \mathrm{~d} x-C(t)\right)^{2}}\right] \mathrm{d} x \\
& =-\int\left(\frac{\int g_{1} \psi_{1, x} \mathrm{~d} x}{\int f_{1, x} g_{1} \mathrm{~d} x-C(t)}\right)_{x} \mathrm{~d} x \\
& =-\frac{\int g_{1} \psi_{1, x} \mathrm{~d} x}{\int f_{1, x} g_{1} \mathrm{~d} x-C(t)} \\
& =\text { the r.h.s of (4.18). }
\end{aligned}
$$


This completes the proof.

Remark: If $C(t)$ is not a constant, i.e. $\frac{d}{d t} C(t) \neq 0$, the DT (4.15) provides a nonauto-Bäcklund transformation between two mKPESCSs (4.6) with degree $N$ and $N+1$ respectively.

\section{The n-times Repeated Generalized Darboux Trans- formation for the mKPESCS}

Assuming $f_{1}, \ldots, f_{n}$ are $n$ arbitrary solutions of (4.7) and $g_{1}, \ldots, g_{n}$ are $\mathrm{n}$ arbitrary solutions of (4.8), $C_{1}(t), \ldots, C_{n}(t)$ are $n$ arbitrary functions in $t$, we define the following Wronskians:

$$
\begin{array}{ll}
W_{1}\left(f_{1}, \ldots, f_{n} ; g_{1}, \ldots, g_{n} ; C_{1}(t), \ldots, C_{n}(t)\right) & =\operatorname{det}\left(X_{n \times n}\right), \\
W_{2}\left(f_{1}, \ldots, f_{n} ; g_{1}, \ldots, g_{n} ; C_{1}(t), \ldots, C_{n}(t)\right) & =\operatorname{det}\left(\tilde{X}_{n \times n}\right), \\
W_{3}\left(f_{1}, \ldots, f_{n} ; g_{1}, \ldots, g_{n-1} ; C_{1}(t), \ldots, C_{n-1}(t)\right) & =\operatorname{det}\left(Y_{n \times n}\right), \\
W_{4}\left(f_{1}, \ldots, f_{n-1} ; g_{1}, \ldots, g_{n} ; C_{1}(t), \ldots, C_{n-1}(t)\right) & =\operatorname{det}\left(\tilde{Y}_{n \times n}\right),
\end{array}
$$

where

$$
\begin{gathered}
X_{i, j}=-\delta_{i, j} C_{i}(t)+\int g_{j} f_{i, x} \mathrm{~d} x \\
\tilde{X}_{i, j}=\delta_{i, j} C_{i}(t)+\int g_{j, x} f_{i} \mathrm{~d} x, \quad i, j=1, \ldots, n, \\
Y_{i, j}=-\delta_{i, j} C_{i}(t)+\int g_{i} f_{j, x} \mathrm{~d} x, i=1, \ldots, n-1, j=1, \ldots, n ; \quad Y_{n, j}=f_{j}, j=1, \ldots, n . \\
\tilde{Y}_{i, j}=\delta_{i, j} C_{i}(t)+\int g_{j, x} f_{i} \mathrm{~d} x, i=1, \ldots, n-1, j=1, \ldots, n ; \quad \tilde{Y}_{n, j}=g_{j}, j=1, \ldots, n .
\end{gathered}
$$

Lemma 5.1 Assume $f_{1}, \ldots, f_{n}$ are solutions of (4.7) and $g_{1}, \ldots, g_{n}$ are solutions of (4.8), then for $2 \leq m \leq n, 1 \leq k \leq n-m$, we have

$$
\begin{aligned}
& W_{1}\left(f_{m}[m-1], \ldots, f_{m+k}[m-1] ; g_{m}[m-1], \ldots, g_{m+k}[m-1] ; C_{m}(t), \ldots, C_{m+k}(t)\right) \\
= & \frac{W_{1}\left(f_{m-1}[m-2], \ldots, f_{m+k}[m-2] ; g_{m-1}[m-2], \ldots, g_{m+k}[m-2] ; C_{m-1}(t), \ldots, C_{m+k}(t)\right)}{-C_{m-1}(t)+\int f_{m-1}[m-2] x g_{m-1}[m-2] \mathrm{d} x}, \\
= & \frac{W_{2}\left(f_{m}[m-1], \ldots, f_{m+k}[m-1] ; g_{m}[m-1], \ldots, g_{m+k}[m-1] ; C_{m}(t), \ldots, C_{m+k}(t)\right)}{} \\
& W_{3}\left(f_{m}[m-1], \ldots, f_{m+k}[m-1] ; g_{m}[m-1], \ldots, g_{m+k}[m-2] ; g_{m-1}[m-2], \ldots, g_{m+k}[m-2] ; C_{m-1}(t), \ldots, C_{m+k}(t)\right) \\
= & \frac{W_{3}\left(f_{m-1}[m-2], \ldots, f_{m+k}[m-2] ; g_{m-1}[m-2], \ldots, g_{m+k-1}[m-2] ; C_{m-1}(t), \ldots, C_{m+k-1}(t)\right)}{-C_{m-1}(t)+\int f_{m-1}[m-2] x g_{m-1}[m-2] \mathrm{d} x}
\end{aligned}
$$




$$
\begin{aligned}
& W_{4}\left(f_{m}[m-1], \ldots, f_{m+k-1}[m-1] ; g_{m}[m-1], \ldots, g_{m+k}[m-1] ; C_{m}(t), \ldots, C_{m+k-1}(t)\right) \\
= & \frac{W_{4}\left(f_{m-1}[m-2], \ldots, f_{m+k-1}[m-2] ; g_{m-1}[m-2], \ldots, g_{m+k}[m-2] ; C_{m-1}(t), \ldots, C_{m+k-1}(t)\right)}{C_{m-1}(t)+\int f_{m-1}[m-2] g_{m-1}[m-2] x} .
\end{aligned}
$$

This lemma can be proved in the same way as we did in [17]. Then we have

Theorem 5.1 Assume that $u, \Psi_{1}, \cdots, \Psi_{N}, \Phi_{1}, \cdots, \Phi_{N}$ is a solution of the mKPESCS (4.6), $f_{1}, \cdots, f_{n}$ and $g_{1}, \cdots, g_{n}$ are solutions of (4.7) and (4.8) respectively, $C_{1}(t), \ldots, C_{n}(t)$ are $n$ arbitrary functions in $t$. Then the $n$-times repeated generalized Darboux transformation for (4.7) is given by

$$
\begin{gathered}
\psi_{1}[n]=\frac{W_{3}\left(f_{1}, \ldots, f_{n}, \psi_{1} ; g_{1}, \ldots, g_{n} ; C_{1}(t), \ldots, C_{n}(t)\right)}{W_{1}\left(f_{1}, \ldots, f_{n} ; g_{1}, \ldots, g_{n} ; C_{1}(t), \ldots, C_{n}(t)\right)} \\
u[n]=u+\partial_{x} \ln \frac{W_{2}\left(f_{1}, \ldots, f_{n} ; g_{1}, \ldots, g_{n} ; C_{1}(t), \ldots, C_{n}(t)\right)}{W_{1}\left(f_{1}, \ldots, f_{n} ; g_{1}, \ldots, g_{n} ; C_{1}(t), \ldots, C_{n}(t)\right)} \\
\Psi_{i}[n]=\frac{W_{3}\left(f_{1}, \ldots, f_{n}, \Psi_{i} ; g_{1}, \ldots, g_{n} ; C_{1}(t), \ldots, C_{n}(t)\right)}{W_{1}\left(f_{1}, \ldots, f_{n} ; g_{1}, \ldots, g_{n} ; C_{1}(t), \ldots, C_{n}(t)\right)} \\
\Phi_{i}[n]=\frac{W_{4}\left(f_{1}, \ldots, f_{n} ; g_{1}, \ldots, g_{n}, \Phi_{i} ; C_{1}(t), \ldots, C_{n}(t)\right)}{W_{2}\left(f_{1}, \ldots, f_{n} ; g_{1}, \ldots, g_{n} ; C_{1}(t), \ldots, C_{n}(t)\right)} \\
\Psi_{N+j}[n] \quad-\frac{1}{2} \sqrt{\dot{C}_{j}(t)} \frac{W_{3}\left(f_{1}, \ldots, f_{j-1}, f_{j+1}, \ldots, f_{n}, f_{j} ; g_{1}, \ldots, g_{j-1}, g_{j+1}, \ldots, g_{n} ; C_{1}(t), \ldots, C_{j-1}(t), C_{j+1}(t), \ldots, C_{n}(t)\right)}{W_{1}\left(f_{1}, \ldots, f_{n} ; g_{1}, \ldots, g_{n} ; C_{1}(t), \ldots, C_{n}(t)\right)} \\
\Phi_{N+j}[n] \\
=\frac{1}{2} \sqrt{\dot{C}_{j}(t)} \frac{W_{4}\left(f_{1}, \ldots, f_{j-1}, f_{j+1}, \ldots, f_{n} ; g_{1}, \ldots, g_{j-1}, g_{j+1}, \ldots, g_{n}, g_{j} ; C_{1}(t), \ldots, C_{j-1}(t), C_{j+1}(t), \ldots, C_{n}(t)\right)}{W_{2}\left(f_{1}, \ldots, f_{n} ; g_{1}, \ldots, g_{n} ; C_{1}(t), \ldots, C_{n}(t)\right)} \\
i=1, \ldots, N, \quad j=1, \ldots, n .
\end{gathered}
$$$$
\Psi_{N+j}[n]
$$$$
\Phi_{N+j}[n]
$$

Namely,

$$
\begin{gathered}
\alpha \Psi_{l}[n]_{y}=\Psi_{l}[n]_{x x}-2 u[n] \Psi_{l}[n]_{x}, \\
\alpha \Phi_{l}[n]_{y}=-\Phi_{l}[n]_{x x}-2 u[n] \Phi_{l}[n]_{x}, \quad l=1, \ldots, N+n, \\
\alpha \psi_{1}[n]_{y}=\psi_{1}[n]_{x x}-2 u[n] \psi_{1}[n], \\
\psi_{1}[n]_{t}=A_{1}(u[n]) \psi_{1}[n]+T_{N+n}^{1}(\Psi[n], \Phi[n]) \psi_{1}[n] .
\end{gathered}
$$

So $u[n], \Psi_{j}[n], \Phi_{j}[n], j=1, \ldots, N+n$ satisfy the $\operatorname{mKPESCS}(4.6)$ with degree $(N+n)$. 
Proof: By (4.15) and (5.3), we have

$$
\begin{aligned}
\psi_{1}[n]= & \frac{W_{3}\left(f_{n}[n-1], \psi_{1}[n-1] ; g_{n}[n-1] ; C_{n}(t)\right)}{W_{1}\left(f_{n}[n-1] ; g_{n}[n-1] ; C_{n}(t)\right)} \\
= & \frac{W_{3}\left(f_{n-1}[n-2], f_{n}[n-2], \psi_{1}[n-2] ; g_{n-1}[n-2], g_{n}[n-2] ; C_{n-1}(t), C_{n}(t)\right)}{-C_{n-1}(t)+\int f_{n-1}[n-2]_{x} g_{n-1}[n-2] \mathrm{d} x} \\
& \times \frac{-C_{n-1}(t)+\int f_{n-1}[n-2]_{x} g_{n-1}[n-2] \mathrm{d} x}{W_{1}\left(f_{n-1}[n-2], f_{n}[n-2] ; g_{n-1}[n-2], g_{n}[n-2] ; C_{n-1}(t), C_{n}(t)\right)} \\
= & \cdots \\
= & \frac{W_{3}\left(f_{1}, f_{2}, \ldots, f_{n}, \psi_{1} ; g_{1}, \ldots, g_{n} ; C_{1}(t), \ldots, C_{n}(t)\right)}{W_{1}\left(f_{1}, \ldots, f_{n} ; g_{1}, \ldots, g_{n} ; C_{1}(t), \ldots, C_{n}(t)\right)} .
\end{aligned}
$$

Similarly we can prove (5.4c) and (5.4d) hold.

$$
\begin{aligned}
& u[n]=u[n-1]+\partial_{x} \ln \frac{W_{2}\left(f_{n}[n-1] ; g_{n}[n-1] ; C_{n}(t)\right)}{W_{1}\left(f_{n}[n-1] ; g_{n}[n-1] ; C_{n}(t)\right)} \\
& =u[n-2]+\partial_{x} \ln \frac{\int f_{n-1}[n-2] g_{n-1}[n-2]_{x} \mathrm{~d} x+C_{n-1}(t)}{\int f_{n-1}[n-2]} \partial_{x} \ln \frac{W_{2}\left(f_{n}[n-1] ; g_{n}[n-1] ; C_{n}(t)\right)}{W_{1}\left(f_{n}[n-1] ; g_{n}[n-1] ; C_{n}(t)\right)}
\end{aligned}
$$

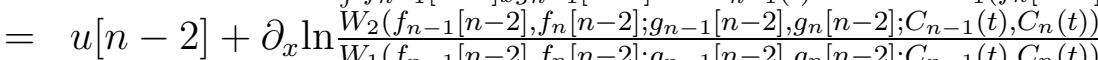

$$
\begin{aligned}
& =\ldots \text {. } \\
& =u+\partial_{x} \ln \frac{W_{2}\left(f_{1}, \ldots, f_{n} ; g_{1}, \ldots, g_{n} ; C_{1}(t), \ldots, C_{n}(t)\right)}{W_{1}\left(f_{1}, \ldots, f_{n} ; g_{1}, \ldots, g_{n} ; C_{1}(t), \ldots, C_{n}(t)\right)} . \\
& f_{j}[j]=f_{j}[j-1]-\frac{f_{j}[j-1] \int g_{j}[j-1] f_{j}[j-1]_{x} \mathrm{~d} x}{\int g_{j}[j-1] f_{j}[j-1]_{x} \mathrm{~d} x-C_{j}(t)}=-\frac{C_{j}(t) f_{j}[j-1]}{\int g_{j}[j-1] f_{j}[j-1]_{x} \mathrm{~d} x-C_{j}(t)}, \\
& \Psi_{N+j}[j]=-\frac{1}{2} \frac{\sqrt{\dot{C}_{j}(t)} f_{j}[j-1]}{\int f_{j}[j-1]_{x} g_{j}[j-1] \mathrm{d} x-C_{j}(t)},
\end{aligned}
$$

SO

$$
\Psi_{N+j}[j]=\frac{1}{2} \frac{\sqrt{\dot{C}_{j}(t)}}{C_{j}(t)} f_{j}[j] .
$$

So

$$
\begin{aligned}
& \Psi_{N+j}[n] \\
= & \frac{W_{3}\left(f_{n}[n-1], \Psi_{N+j}[n-1] ; g_{n}[n-1] ; C_{n}(t)\right)}{W_{1}\left(f_{n}[n-1] ; g_{n}[n-1] ; C_{n}(t)\right)} \\
= & \cdots \\
= & \frac{W_{3}\left(f_{j+1}[j], \cdots, f_{n}[j], \Psi_{N+j}[j] ; g_{j+1}[j], \cdots, g_{n}[j] ; C_{j+1}(t), \cdots, C_{n}(t)\right)}{W_{1}\left(f_{j+1}[j], \cdots, f_{n}[j] ; g_{j+1}[j], \cdots, g_{n}[j] ; C_{j+1}(t), \cdots, C_{n}(t)\right)} \\
= & \frac{\sqrt{\dot{C}_{j}(t)}}{2 C_{j}(t)} \frac{W_{3}\left(f_{j+1}[j], \cdots, f_{n}[j], f_{j}[j] ; g_{j+1}[j], \cdots, g_{n}[j] ; C_{j+1}(t), \cdots, C_{n}(t)\right)}{W_{1}\left(f_{j+1}[j], \cdots, f_{n}[j] ; g_{j+1}[j], \cdots, g_{n}[j] ; C_{j+1}(t), \cdots, C_{n}(t)\right)} \\
= & \cdots \\
= & \frac{\sqrt{\dot{C}_{j}(t)}}{2 C_{j}(t)} \frac{W_{3}\left(f_{1}, \cdots, f_{n}, f_{j} ; g_{1}, \cdots, g_{n} ; C_{1}(t), \cdots, C_{n}(t)\right)}{W_{1}\left(f_{1}, \cdots, f_{n} ; g_{1}, \cdots, g_{n} ; C_{1}(t), \cdots, C_{n}(t)\right)} \\
= & -\frac{1}{2} \sqrt{\dot{C}_{j}(t)} \frac{W_{3}\left(f_{1}, \ldots, f_{j-1}, f_{j+1}, \ldots, f_{n}, f_{j} ; g_{1}, \ldots, g_{j-1}, g_{j+1}, \ldots, g_{n} ; C_{1}(t), \ldots, C_{j-1}(t), C_{j+1}(t), \ldots, C_{n}(t)\right)}{W_{1}\left(f_{1}, \ldots, f_{n} ; g_{1}, \ldots, g_{n} ; C_{1}(t), \ldots, C_{n}(t)\right)} .
\end{aligned}
$$


Similarly we can prove (5.4f) holds.

This completes the proof.

Remark: If $C_{j}(t), j=1, \ldots, n$ are not constants, i.e. $\frac{d}{d t} C_{j}(t) \neq 0$, the DT (5.4) provides a non-auto-Bäcklund transformation between two mKPESCSs (4.6) with degree $N$ and $N+n$ respectively.

\section{Some examples of solutions for the mKPESCS}

\section{Rational solution.}

Example 1: Rational solution with singularities for the mKPIIESCS $(\alpha=1)$. If we set $\alpha=1$ in equation (4.6), we get the mKPIIESCS

$$
\begin{gathered}
u_{t}+u_{x x x}+3 D^{-1}\left(u_{y y}\right)-6 D^{-1}\left(u_{y}\right) u_{x}-6 u^{2} u_{x}+4 \sum_{i=1}^{N}\left(\Psi_{i} \Phi_{i}\right)_{x}=0, \\
\Psi_{i, y}=\Psi_{i, x x}-2 u \Psi_{i, x} \\
\Phi_{i, y}=-\Phi_{i, x x}-2 u \Phi_{i, x} .
\end{gathered}
$$

We take $u=0, \Phi_{1}=a e^{k x-k^{2} y}, \Psi_{1}=b e^{-k x+k^{2} y}, k, a, b \in \mathbb{R}$ as the initial solution of (6.1) with $N=1$ and let

$$
f_{1}=\left(2 x+8 k y-96 k^{2} t-\frac{8 a b t}{9 k}\right) e^{2 k x+4 k^{2} y-32 k^{3} t-\frac{8 a b}{3} t}, \quad g_{1}=e^{2 k x-4 k^{2} y-32 k^{3} t+8 a b t}, \quad C(t)=0,
$$

then by DT (4.15), we get the rational solution with singularities for the mKPIIESCS (6.1) with $N=1$ as follows

$$
\begin{gathered}
u[1]=\partial_{x} \ln \frac{\int f_{1} g_{1, x} \mathrm{~d} x}{\int g_{1} f_{1, x} \mathrm{~d} x}=\frac{8 k}{(2 k A+1)(2 k A-1)}, \\
\Psi_{1}[1]=3 b e^{-k x+k^{2} y} \frac{A+\frac{1}{6 k}}{A+\frac{1}{2 k}} \\
\Phi_{1}[1]=\frac{1}{3} a e^{k x-k^{2} y} \frac{A-\frac{1}{6 k}}{A-\frac{1}{2 k}}
\end{gathered}
$$

where $A=2 x+8 k y-96 k^{2} t-\frac{8 a b t}{9 k}$.

More generally, if we take

$$
f_{i}=\left(x+2 k_{i} y-12 k_{i}^{2} t+\frac{4 a b t}{\left(k_{i}+k\right)^{2}}\right) e^{k_{i} x+k_{i}^{2} y-4 k_{i}^{3}-\frac{4 a b t}{k_{i}+k}}
$$




$$
\begin{gathered}
g_{i}=e^{k_{i} x-k_{i}^{2} y-4 k_{i}^{3}+\frac{4 k_{i} a b t}{k_{i}-k}} \\
C_{i}(t)=0, \quad k_{i} \neq \pm k, i=1, \ldots, n, k_{i}+k_{j} \neq 0, \forall i, j,
\end{gathered}
$$

then $(5.4 \mathrm{~b}),(5.4 \mathrm{c})$ and $(5.4 \mathrm{~d})$ will give the rational solution with multi-singularities for the mKPIIESCS with $N=1$.

Example 2: Lump solution for the mKPIESCS $(\alpha=i)$.

If we set $\alpha=i$ in equation (4.6), we get the mKPIESCS

$$
\begin{gathered}
u_{t}+u_{x x x}-3 D^{-1}\left(u_{y y}\right)-6 i D^{-1}\left(u_{y}\right) u_{x}-6 u^{2} u_{x}+4 \sum_{i=1}^{N}\left(\Psi_{i} \Phi_{i}\right)_{x}=0 \\
i \Psi_{i, y}=\Psi_{i, x x}-2 u \Psi_{i, x} \\
i \Phi_{i, y}=-\Phi_{i, x x}-2 u \Phi_{i, x} .
\end{gathered}
$$

We take $u=0, \Phi_{1}=a e^{-i k x-i k^{2} y}, \Psi_{1}=b e^{i k x+i k^{2} y}, k, a, b \in \mathbb{R}$ as the initial solution of (6.4) with $N=1$ and let

$$
f_{1}=\left(x-2 l y+12 l^{2} t-\frac{4 a b k t i}{(k+l)^{2}}\right) e^{-i l x+i l^{2} y-4 i l^{3} t-\frac{4 a b l t}{k+l}}, \quad g_{1}=e^{-i l x-i l^{2} y-4 i l^{3} t+\frac{4 a b l t}{l-k}},
$$

$l \in \mathbb{R}, l \neq \pm k$ and $C(t)=0$, then by DT (4.15), we get the 1-lump solution for the mKPIESCS (6.4) with $N=1$ as follows

$$
\begin{gathered}
u[1]=\partial_{x} \ln \frac{\int f_{1} g_{1, x} \mathrm{~d} x}{\int g_{1} f_{1, x} \mathrm{~d} x}=\frac{4 l i}{1+(2 l A)^{2}}, \\
\Psi_{1}[1]=b e^{k x i+k^{2} y i} \frac{-2 A l(k+l)+i(k-l)}{2 l A(k-l)+i(k-l)}, \\
\Phi_{1}[1]=a e^{-k x i-k^{2} y i} \frac{2 l A\left(k^{2}-l^{2}\right)+(k-l)^{2} i}{-2 l A(l+k)^{2}+i(k+l)^{2}},
\end{gathered}
$$

where $A=x-2 l y+12 l^{2} t-4 a b \frac{i k t}{(k+l)^{2}}$.

More generally, if we take

$$
\begin{gathered}
f_{j}=\left(x-2 l_{j} y+12 l_{j}^{2} t+\frac{4 l_{j} a b t}{\left(l_{j}+k\right)^{2}}\right) e^{-i l_{j} x+i l_{j}^{2} y-4 i l_{j}^{3} t-\frac{4 i l_{j} a b t}{l_{j}+k}} \\
g_{j}=e^{-i l_{j} x-i l_{j}^{2} y-4 i l_{j}^{3} t+\frac{4 i l_{j} a b t}{l_{j}-k}} \\
C_{j}(t)=0, \quad l_{j} \neq \pm k, j=1, \ldots, n, l_{m}+l_{j} \neq 0, \forall m, j
\end{gathered}
$$


then (5.4b), (5.4c) and (5.4d) will give the multi-lump solution for the mKPIESCS with $N=1$.

\section{Soliton solution.}

Example 3: Soliton solution for the mKPIIESCS.

We take $u=0$ as the initial solution for the mKPIIESCS (6.1) with $N=0$ and let

$$
f_{1}=e^{k x+k^{2} y-4 k^{3} t}=e^{\xi_{1}}, \quad g_{1}=e^{l x-l^{2} y-4 l^{3} t}=e^{\xi_{2}}, \quad C(t)=e^{2 \beta(t)},
$$

where $k, l \in \mathbb{R}, k+l \neq 0$, and $\beta(t)$ is an arbitrary function in $t$. Then by DT (4.15), we get the 1-soliton solution for the mKPIIESCS (6.1) with $N=1$ as follows

$$
\begin{gathered}
u[1]=\partial_{x} \ln \frac{\int f_{1} g_{1, x} \mathrm{~d} x+C(t)}{\int g_{1} f_{1, x} \mathrm{~d} x-C(t)}=-\frac{k+l}{\left(\frac{l}{k+l} e^{\eta}-e^{-\eta}\right)\left(\frac{k}{k+l} e^{\eta}+e^{-\eta}\right)}, \quad \eta=\frac{\xi_{1}+\xi_{2}}{2}-\beta(t), \\
\Psi_{1}[1]=-\frac{1}{2} f_{1} \frac{\sqrt{\dot{C}(t)}}{\int f_{1, x} g_{1} \mathrm{~d} x-C(t)}=-\frac{\sqrt{2 \dot{\beta}(t)}}{2} \frac{e^{\xi_{1}+\beta(t)}}{\frac{k}{k+l} e^{\xi_{1}+\xi_{2}}-e^{2 \beta(t)}}, \\
\Phi_{1}[1]=\frac{1}{2} g_{1} \frac{\sqrt{\dot{C}(t)}}{\int g_{1, x} f_{1} \mathrm{~d} x+C(t)}=\frac{\sqrt{2 \dot{\beta}(t)}}{2} \frac{e^{\xi_{2}+\beta(t)}}{\frac{l}{k+l} e^{\xi_{1}+\xi_{2}}+e^{2 \beta(t)}} .
\end{gathered}
$$

More generally, if we take

$$
f_{i}=e^{k_{i} x+k_{i}^{2} y-4 k_{i}^{3} t}, \quad g_{i}=e^{l_{i} x-l_{i}^{2} y-4 l_{i}^{3} t}, \quad C_{i}(t)=e^{\beta_{i}(t)}, i=1, \ldots, n,
$$

where $k_{i}, l_{i} \in \mathbb{R}, k_{i}+l_{j} \neq 0, \forall i, j$, then (5.4b), (5.4e) and (5.4f) will give the n-soliton solution for the mKPIIESCS with $N=n$.

Example 4: Soliton solution for the mKPIESCS.

We take $u=0$ as the initial solution for the mKPIESCS (6.4) with $N=0$ and let

$$
f_{1}=e^{-i k x+i k^{2} y-4 i k^{3} t}, \quad g_{1}=e^{i \bar{k} x-i \bar{k}^{2} y+4 i \bar{k}^{3} t}, \quad C(t)=i e^{2 \beta(t)}
$$

where $k \in \mathbb{C}$ and $\beta(t)$ is an arbitrary function in $t$.

Set $k=\mu-i \nu, \quad \mu, \nu \in \mathbb{R}, \quad \nu \neq 0$,

then

$$
f_{1}=e^{\theta+\eta}, \quad g_{1}=\bar{f}_{1}=e^{-\theta+\eta}
$$

where

$$
\theta=-i \mu x+i\left(\mu^{2}-\nu^{2}\right) y-4 i\left(\mu^{3}-3 \mu \nu^{2}\right) t, \quad \eta=-\nu x+2 \mu \nu y+4 \nu\left(\nu^{2}-3 \mu^{2}\right) t .
$$


Then by DT (4.15), we get the 1-soliton solution for the mKPIESCS (6.4) with $N=1$ as follows

$$
\begin{gathered}
u[1]=\partial_{x} \ln \frac{\int f_{1} g_{1, x} \mathrm{~d} x+C(t)}{\int g_{1} f_{1, x} \mathrm{~d} x-C(t)}=\frac{2 \nu i}{\frac{1}{4} e^{2 f}+\left(e^{-f}-\frac{\mu}{2 \nu} e^{f}\right)^{2}}, \quad f=\eta-\beta(t) \\
\Psi_{1}[1]=-\frac{1}{2} f_{1} \frac{\sqrt{\dot{C}(t)}}{\int f_{1, x} g_{1} \mathrm{~d} x-C(t)}=\frac{\sqrt{\dot{\beta}(t)}(1-i) e^{\theta+\nu x+2 \mu \nu y+4 \nu^{3} t+12 \mu^{2} \nu t}}{(i \nu-\mu) e^{8 \nu^{3} t+4 \mu \nu y}+2 \nu e^{\beta(t)+24 \mu^{2} \nu t+2 \nu x}} \\
\Phi_{1}[1]=\frac{1}{2} g_{1} \frac{\sqrt{\dot{C}(t)}}{\int g_{1, x} f_{1} \mathrm{~d} x+C(t)}=\frac{\sqrt{\dot{\beta}(t)}(i-1) e^{-\theta+\nu x+2 \mu \nu y+4 \nu^{3} t+12 \mu^{2} \nu t}}{(i \nu+\mu) e^{8 \nu^{3} t+4 \mu \nu y}-2 \nu e^{\beta(t)+24 \mu^{2} \nu t+2 \nu x}}
\end{gathered}
$$

More generally, if we take

$$
f_{j}=e^{-i k_{j} x+i k_{j}^{2} y-4 i k_{j}^{3} t}, \quad g_{j}=e^{i \bar{k}_{j} x-i \bar{k}_{j}^{2} y+4 i \bar{k}_{j}^{3} t}, \quad C_{j}(t)=i e^{2 \beta_{j}(t)}, j=1, \ldots, n,
$$

where $k_{j}=\mu_{j}+i \nu_{j}, \mu_{j}, \nu_{j} \in \mathbb{R}, k_{j} \neq \bar{k}_{m}, \forall j, m$ and $\beta_{j}(t), j=1, \ldots, n$, are arbitrary functions in $t$, then $(5.4 \mathrm{~b}),(5.4 \mathrm{e})$ and $(5.4 \mathrm{f})$ will give the n-soliton solution for the mKPIESCS with $N=n$.

\section{Solutions of breather type.}

Example 5: Solutions of breather type for the mKPIESCS.

We take $u=0$ as the initial solution for the mKPIESCS (6.4) with $N=0$. If we take

$$
f_{j}=e^{-i \lambda_{j} x+i \lambda_{j}^{2} y-4 i \lambda_{j}^{3} t}, \quad g_{j}=e^{i \xi_{j} x-i \xi_{j}^{2} y+4 i \xi_{j}^{3} t}, \quad C_{j}(t)=i e^{2 \beta_{j}(t)}, \quad j=1, \ldots, 2 n
$$

where

$$
\left(\lambda_{1}, \ldots, \lambda_{2 n}\right)=\left(k_{1}, \ldots, k_{n} ; l_{1}, \ldots, l_{n}\right), \quad\left(\xi_{1}, \ldots, \xi_{2 n}\right)=\left(\bar{l}_{1}, \ldots, \bar{l}_{n} ; \bar{k}_{1}, \ldots, \bar{k}_{n}\right),
$$

$k_{j}, l_{j} \in \mathbb{C}, \operatorname{Im}\left(k_{j}\right) \neq 0, \operatorname{Im}\left(l_{j}\right) \neq 0, l_{m} \neq \bar{k}_{j}, \forall m, j$,

we will get the solutions of breather type for the mKPIESCS by (5.4b), (5.4e) and (5.4f).

For example, if we choose $n=1$ and

$$
\lambda_{1}=k_{1}=-b i, \quad \lambda_{2}=l_{1}=-d i, \quad \xi_{1}=\bar{\lambda}_{2}=d i, \quad \xi_{2}=\bar{\lambda}_{1}=b i, \quad C_{1}(t)=C_{2}(t)=i e^{2 t},
$$

we will get the following solution of mKPIESCS

$$
\begin{gathered}
u[2]=-\frac{8(b+d)^{2} \cos \theta \mathrm{e}^{\mathrm{f}} \mathrm{i}}{4(b+d)^{2} e^{2 t}+4\left(b^{2}-d^{2}\right) e^{f}+(b-d)^{2} e^{2 f-2 t}}, \\
\Psi_{1}[2]=\frac{1+i}{2} e^{\eta_{1}+\theta_{2} i+t} \frac{\frac{d-b}{2(d+b)} e^{f}+e^{2 t} \sin \theta-\mathrm{ie}^{2 \mathrm{t}} \cos \theta}{e^{4 t}-e^{f+2 t} \sin \theta \frac{\mathrm{b}-\mathrm{d}}{\mathrm{b}+\mathrm{d}}+\frac{(\mathrm{b}-\mathrm{d})^{2}}{4(\mathrm{~b}+\mathrm{d})^{2}} \mathrm{e}^{2 \mathrm{t}}+\mathrm{e}^{\mathrm{f}+2 \mathrm{t}} \cos \theta \mathrm{i}}
\end{gathered}
$$




$$
\begin{gathered}
\Psi_{2}[2]=\frac{1+i}{2} e^{\eta_{2}+\theta_{1} i+t} \frac{\frac{b-d}{2(d+b)} e^{f}-e^{2 t} \sin \theta-\mathrm{ie}^{2 \mathrm{t}} \cos \theta}{e^{4 t}-e^{f+2 t} \sin \theta \frac{\mathrm{b}-\mathrm{d}}{\mathrm{b}+\mathrm{d}}+\frac{(\mathrm{b}-\mathrm{d})^{2}}{4(\mathrm{~b}+\mathrm{d})^{2}} \mathrm{e}^{2 \mathrm{t}}+\mathrm{e}^{\mathrm{f}+2 \mathrm{t}} \cos \theta \mathrm{i}}, \\
\Phi_{1}[2]=-\frac{1+i}{2} e^{\eta_{2}-\theta_{1} i+t} \frac{\frac{b+d}{2(b-d)} e^{f}-e^{2 t} \sin \theta+\mathrm{e}^{2 \mathrm{t}} \cos \theta}{e^{4 t}-e^{f+2 t} \sin \theta \frac{\mathrm{b}-\mathrm{d}}{\mathrm{b}+\mathrm{d}}+\frac{(\mathrm{b}-\mathrm{d})^{2}}{4(\mathrm{~b}+\mathrm{d})^{2}} \mathrm{e}^{2 \mathrm{t}}-\mathrm{e}^{\mathrm{f}+2 \mathrm{t}} \cos \theta \mathrm{i}}, \\
\Phi_{2}[2]=-\frac{1+i}{2} e^{\eta_{1}-\theta_{2} i+t} \frac{\frac{b+d}{2(d-b)} e^{f}+e^{2 t} \sin \theta+\mathrm{ie}^{2 \mathrm{t}} \cos \theta}{e^{4 t}-e^{f+2 t} \sin \theta \frac{\mathrm{b}-\mathrm{d}}{\mathrm{b}+\mathrm{d}}+\frac{(\mathrm{b}-\mathrm{d})^{2}}{4(\mathrm{~b}+\mathrm{d})^{2}} \mathrm{e}^{2 \mathrm{t}}-\mathrm{e}^{\mathrm{f}+2 \mathrm{t}} \cos \theta \mathrm{i}},
\end{gathered}
$$

where

$$
\begin{gathered}
f=-(b+d) x+4\left(b^{3}+d^{3}\right) t, \quad \theta=\left(d^{2}-b^{2}\right) y, \quad \eta_{1}=-b x+4 b^{3} t, \quad \eta_{2}=-d x+4 d^{3} t, \\
\theta_{1}=-b^{2} y, \quad \theta_{2}=-d^{2} y .
\end{gathered}
$$

$u[2]$ is periodic in $y$ and has soliton behavior along the coordinate $x$. Similarly, we can get the solution of breather type for the mKPIIESCS.

Remark: In Example 1 and Example 2, when $a=b=0$, the solutions obtained above will degenerate to the solutions of the corresponding mKP equations respectively. In Example 3, Example 4 and Example 5, when $C(t)\left(C_{j}(t)\right)$ are taken to be constant(s), i.e. $\frac{d}{d t} C(t)\left(\frac{d}{d t} C_{j}(t)\right)=0$, the solutions obtained above will also degenerate to the solutions of the corresponding mKP equations respectively [24].

\section{Acknowledgment}

This work was supported by the Chinese Basic Research Project "Nonlinear Science".

\section{References}

[1] V.K.Mel'nikov, Integration method of the Korteweg-de Vries equation with a selfconsistent source, Phys. Lett. A 133(1988) 493-496.

[2] V.K.Mel'nikov, Capture and confinement of solitons in nonlinear integrable systems, Commun. Math. Phys. 120 (1989) 451-468.

[3] V.K.Mel'nikov, New method for deriving nonlinear integrable system, J. Math. Phys. 31 (1990) 1106. 
[4] J.Leon, A.Latifi, Solutions of an initial-boundary value problem for coupled nonlinear waves, J. Phys. A 23 (1990) 1385-1403.

[5] J.Leon, Nonlinear evolutions with singular dispersion laws and forced systems, Phys. Lett. A 144 (1990) 444-452.

[6] E.V.Doktorov, R.A.Vlasov, Opt. Acta 30 (1991) 3321.

[7] V.K.Mel'nikov, Integration of the nonlinear Schrödinger equation with a source, Inverse Problems 8 (1992) 133-147.

[8] C.Claude, A.Latifi, J.Leon, Nonliear resonant scattering and plasma instability: an integrable model, J. Math. Phys. 32 (1991) 3321-3330.

[9] Y.B.Zeng, W.X. Ma, R.L.Lin, Integration of the soliton hierarchy with self-consistent sources, J. Math. Phys. 41 (2000) 5453-5489.

[10] R.L.Lin, Y.B.Zeng, W.X.Ma, Solving the KdV hierarchy with self-consistent sources by inverse scattering method, Physica A 291 (2001) 287-298.

[11] S.Ye, Y.B.Zeng, Integration of the mKdV hierarchy with integral type of source, J. Phys. A 35 (2002) 283-291.

[12] Y.B.Zeng, W.X.Ma, Y.J.Shao, Two binary Darboux transformations for the KdV hierarchy with self-consistent sources, J. Math. Phys. 42(5) (2001) 2113-2128.

[13] Y.B.Zeng, Y.J.Shao, W.X.Ma, Integral-type Darboux transformations for the mKdV hierarchy with self-consistent sources, Commun. Theor. Phys. 38 (2002) 641-648.

[14] Y.B.Zeng, Y.J.Shao, and W.M.Xue, Negaton and Positon solutions of the soliton equation with self-consistent sources, J. Phys. A 36 (2003) 1-9.

[15] V.K.Mel'nikov, A direct method for deriving a Multi-soliton solution for the problem of interaction of waves on the x,y plane, Commun. Math. Phys. 112 (1987) 639-652.

[16] V.K.Mel'nikov, Interaction of solitary waves in the system described by the KadomtsevPetviashvili equation with a self-consistent source, Commun. Math. Phys. 126 (1989) 201-215.

[17] T.Xiao, Y.B.Zeng, Generalized Darboux transformations for the KP equation with self-consistent sources, J. Phys. A 37 (2004) 7143-7162. 
[18] S.F.Deng, D.Y.Chen, and D.J.Zhang, The Multisoliton Solutions of the KP Equation with Self-consistent Sources, J. Phys. Soc. Jap. 72(2003) 2184-2192.

[19] W.Oevel, W.Strampp, Constrained KP Hierarchy and Bi-Hamiltonian Structures, Commun. Math. Phys. 157 (1993) 51-81.

[20] P.G.Estévez, P.R.Gordoa, Darboux transformations via Painleve analysis, Inverse Problem 13 (1997) 939-957.

[21] H.H.Chen, Y.C.Lee, C.S.Liu, Integrability of Nonlinear Hamilltonian Systems by Inverse Scattering Method, Phys.Scr. 20 (1979) 490-492.

[22] Y.Cheng, Y.S.Li, Constraints of the $2+1$ dimensional integrable soliton systems, J.Phys.A 25 (1992) 419-431.

[23] X.G.Geng, Y.T.Wu, C.W.Cao, Quasi-periodic solutions of the modified KadomtsevPetviashvili equation, J.Phys.A 32 (1999) 3733-3742.

[24] B.G.Konopelchenko, Inverse Spectral Transform for the Modified KadomtsevPetriashvili Equation, Stud. Appl. Math. 86 (1992) 219-268.

[25] M.J.Ablowitz, P.A.Clarkson, Solitons, Nonlinear Evolution Equations and Inverse Scattering, Cambridge, 1991.

[26] L.A.Dickey, Soliton equation and Hamiltonian systems, World Scientific,Singapore, 1991.

[27] V.B.Matveev, M.A.Salle, Darboux Transformations and Solitons, Springer, Berlin, 1991.

[28] E.Date, M.Jimbo, M.Kashiwara, T.Miwa, In Nonlinear Integrable Systems-Classical Theory and Quantum Theory, 1983; M.Jimbo, T.Miwa (eds.) World Scientific, Singapore, 1983.

[29] Y.Ohta, J.Satsuma, D.Takahashi, T.Tokihiro, An Elementary Introduction to Sato Theory, Prog. Theor. Phys. Suppl. 94 (1988) 210.

[30] J. Sidorenko, W. Strampp, Symmetry constraints of the KP hierarchy, Inverse Probl. 7 (1991) L37-L43.

[31] L.A.Dickey, On the Constrained KP Hierarchy, Lett. Math. Phys. 34 (1995) 379-384. 
[32] Y.Cheng, Constraints of the Kadomtsev-Petriashvili hierarchy, J. Math. Phys. 33 (1992) 3774 .

[33] Y.Cheng, Modifying the KP, the nth Constrained KP Hierarchies and their Hamiltonian Structure, Commun. Math. Phys. 171 (1995) 661-682. 